\title{
Roles for the gut microbiota in regulating neuronal feeding circuits
}

\author{
Kristie B. Yu and Elaine Y. Hsiao \\ Department of Integrative Biology \& Physiology, University of California, Los Angeles, California, USA
}

\begin{abstract}
The gut microbiota has the capacity to affect host appetite via intestinal satiety pathways, as well as complex feeding behaviors. In this Review, we highlight recent evidence that the gut microbiota can modulate food preference across model organisms. We discuss effects of the gut microbiota on the vagus nerve and brain regions including the hypothalamus, mesolimbic system, and prefrontal cortex, which play key roles in regulating feeding behavior. Crosstalk between commensal bacteria and the central and peripheral nervous systems is associated with alterations in signaling of neurotransmitters and neuropeptides such as dopamine, brain-derived neurotrophic factor (BDNF), and glucagon-like peptide-1 (CLP-1). We further consider areas for future research on mechanisms by which gut microbes may influence feeding behavior involving these neural pathways. Understanding roles for the gut microbiota in feeding regulation will be important for informing therapeutic strategies to treat metabolic and eating disorders.
\end{abstract}

\section{Introduction}

Understanding the biological bases of feeding behaviors is key to developing treatments for increasingly prevalent metabolic and eating disorders, including obesity and anorexia nervosa (1-3). Neurobiological regulation of feeding behavior is extremely complex, involving both energy homeostasis and motivational processes (1-6). Neural pathways that regulate feeding behavior have accordingly been divided into homeostatic and non-homeostatic controls $(1,6)$. Homeostatic controls respond to energy and other metabolic deficits, and classically involve the hypothalamus and brainstem nuclei $(1,2)$. Non-homeostatic controls involve hedonic and cognitive aspects of feeding that are processed by higher-order brain structures including frontal cortical regions, mesolimbic circuitry, and hippocampus $(1,6)$. Blurring this artificial division, neural substrates in either category may interact, and both respond to energy status cues and modulate learned feeding behaviors (1). The vagus nerve, which conducts information bidirectionally between the brain and viscera including the gastrointestinal tract, also connects homeostatic and non-homeostatic feeding regulation by communicating gastrointestinal hunger and satiety signals while modulating higher-order brain regions (7-10).

Adding to this already complex system, ingested nutrients also fuel the trillions of microorganisms that inhabit the host gastrointestinal tract, collectively known as the gut microbiota (11, 12). It logically follows that gut microbes may influence host feeding behavior to promote their own fitness $(11,12)$. Foundational studies in which germ-free mice showed increased body fat after colonization with microbiotas from obese mice or humans compared with their lean counterparts demonstrated a key role for the

Conflict of interest: The authors have declared that no conflict of interest exists. Copyright: (5) 2021, American Society for Clinical Investigation.

Reference information: J Clin Invest. 2021;131(10):e143772.

https://doi.org/10.1172/JCl143772. gut microbiota in the development of obesity $(13,14)$. Since then, the gut microbiota has been increasingly appreciated as regulating host metabolism (15-19) and appetite (11, 20), with translational implications for both obesity $(6,21-23)$ and eating disorders (2427). Microbiota-derived metabolites and bacterial components can influence host appetite via intestinal satiety pathways $(11,20)$. Despite their known effects on host brain and behavior through the microbiota-gut-brain axis (28-30), the mechanisms by which gut microbes influence feeding behavior, such as nutrient preference or food cravings, remain unclear $(12,13)$.

This article reviews recent literature reporting effects of the gut microbiota on brain regions involved in homeostatic and non-homeostatic controls (Figure 1 and Tables 1 and 2) and on the vagus nerve (Figure 2). It further explores areas for future research on potential mechanisms by which gut microbes may influence host feeding behavior.

\section{Gut microbial effects on food preference}

The gut microbiota can influence host dietary preference across animal models $(12,20)$. Recent invertebrate studies demonstrated that microbially derived metabolites modulate host feeding behavior, providing potential insight into similar pathways in higher organisms. Colonization of Caenorhabditis elegans with the commensal bacterium Providencia alters host olfactory behavior in response to a volatile repellent called octanol through the production of tyramine (31). Host enzymes convert bacterially produced tyramine to octopamine, which acts on receptors on octanol-sensing nociceptive neurons, resulting in decreased host aversion to octanol and preference for Providencia in food choice assays (31). Similarly to tyramine, bacterially produced lactate can alter host feeding decisions in Drosophila melanogaster (32). Colonization of flies with the commensal bacteria Acetobacter pomorum and Lactobacillus plantarum suppresses yeast appetite of the host when it is deprived of essential amino acids $(32,33)$. A. pomorum uses lactate produced by $L$. plantarum to synthesize unidentified metabolites 


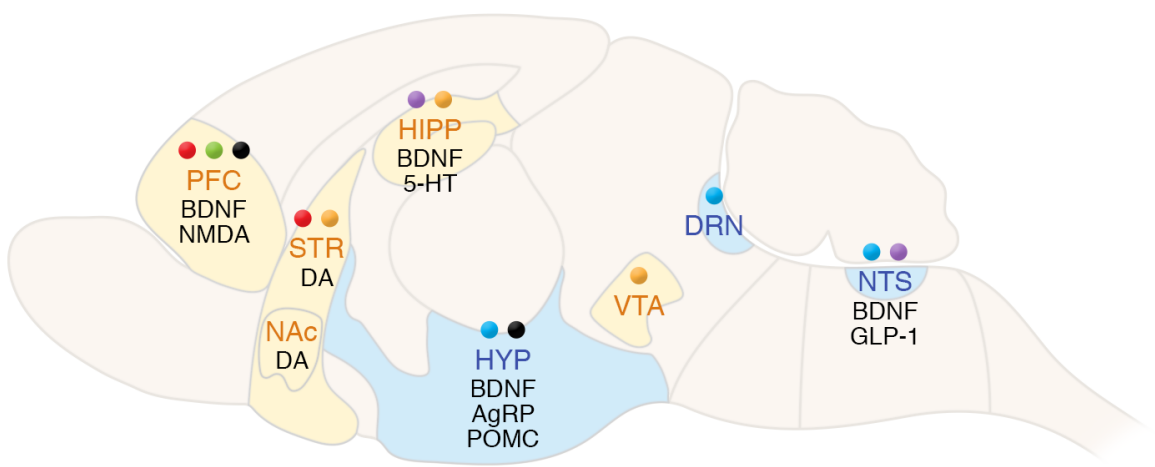

Homeostatic

Nonhomeostatic

Gut microbiota-mediated changes in:

\begin{tabular}{ll}
\hline - AA metabolism & Microglia \\
cFos or $\Delta$ FosB expression & Synapses \\
- Myelination & Transcriptome
\end{tabular}

Figure 1. Gut microbiota-mediated changes in homeostatic and non-homeostatic neural substrates. See Tables 1 and 2 for more detailed information. AA, amino acid; ABX, antibiotics; AgRP, agouti-related peptide; BDNF, brain-derived neurotrophic factor; CART, cocaine- and amphetamine-regulated transcript; CIpB, caseinolytic protease B; CONV-R, conventionally raised; DRN, dorsal raphe nucleus; FOS, fructo-oligosaccharides; GABA, $\gamma$-aminobutyric acid; GF, germ free; GLP-1, glucagon-like peptide-1; GOS, galacto-oligosaccharides; HFD, high-fat diet; HFHS, high-fat, highsugar; HIPP, hippocampus; 5-HT, serotonin; HYP, hypothalamus; LPS, lipopolysaccharide; NAc, nucleus accumbens; NGFI-A, nerve growth factor-inducible clone A; NMDA, $N$-methyl-D-aspartate; NTS, nucleus of the solitary tract; PFC, prefrontal cortex; POMC, pro-opiomelanocortin; PSD-95, postsynaptic density protein 95; STR, striatum; VTA, ventral tegmental area.

\section{Gut microbiota and homeostatic feeding controls}

Hypothalamus. The hypothalamus is critical for maintaining energy homeostasis by integrating environmental, sensory, hormonal, and gastrointestinal nutrient signals to regulate feeding behavior $(1,6$, 8). In particular, two cell populations of the arcuate nucleus (ARC) work antagonistically to control feeding: the anorexigenic (appetite-reducing) pro-opiomelanocortin-expressing (POMC-expressing) neurons and the orexigenic (appetite-stimulating) agouti-related protein/neuropeptide Y-coexpressing (AgRP/NPY-coexpressing) neurons $(1,42)$. Beyond these neurons, the hypothalamus exhibits functional connectivity to many brain regions to enable coordinated cellular responses to internal metabolic states (43-45).

The gut microbiota's key role in host metabolism (15-19) has raised the question of whether it may modify hypothalamic activity. Increases in the taxonomic diversity of the gut microbiota significantly correlate with sparing of hypothalamic brain microstructure in obese and nonobese

that are required to alter host food choice (32). Together, these studies suggest that molecules produced from both host-bacterial and bacterial-bacterial metabolic interactions can modify host sensory and feeding behavior.

In mammals such as mice, a few correlational studies suggest that the microbiota can affect host taste and thus food choice (12, 20,34 ). Compared with mice with a conventional microbiota (conventionally raised [CONV-R]), mice raised in the absence of the gut microbiota (germ free [GF]) show increased sucrose intake and upregulation of intestinal sweet taste receptors and glucose transporter (35). The type 2 family of taste receptors (T2Rs), which mediate bitter taste, are activated by bacterial signaling molecules in the respiratory tract (34). Since T2Rs are also expressed in the intestine and regulate GI functions, bacterial interactions with these receptors have been hypothesized but remain to be proven. Although these taste receptors are extraoral $(34,35)$, they impact food intake (36) and open the possibility for microbial influence on oral taste. A recent study showed that prebiotic treatment with inulin-type fructans can affect host sweet taste perception in mice (37). Prebiotic treatment partially corrects blunted sweet taste perception in diet-induced obese mice by increasing sucrose preference and also ameliorates gut microbiota dysbiosis, suggesting a correlation between taste and gut microbiota composition (37). In addition to taste, endocannabinoid signaling (38-40) may be another possible avenue for gut microbes to affect host food preference (41). Future studies are needed to evaluate causal effects of the gut microbiota on host taste and food preference in mammals. These include assessing whether microbial molecules can interact with oral and intestinal taste receptors and identifying specific bacteria that can modulate food preference in GF mice. individuals (46), suggesting that the gut microbiota may modulate hypothalamic function in humans. Supporting this notion, several animal studies have demonstrated the capacity of the gut microbiota to alter hypothalamic gene expression, neuropeptide and neurotransmitter levels, and neuronal activity. GF mice exhibit altered hypothalamic expression of feeding-related neuropeptides compared with CONV-R mice $(47,48)$. In one study, CONV-R mice showed decreased expression of the anorexigenic neuropeptide brain-derived neurotrophic factor $(B d n f)$ in the hypothalamus compared with GF mice, which potentially contributes to fat mass induction by the gut microbiota (47). Secondary to elevated fat mass, CONV-R mice show a compensatory decrease in the expression of orexigenic Npy and Agrp, increase in anorexigenic Pomc and Cart, and increase in leptin resistance-associated Socs-3 compared with GF mice (47). In contrast, another study found that GF mice show higher hypothalamic Pomc and Socs-3 expression than CONV-R mice (48). This discrepancy can potentially be explained by differences in diet composition and other rearing conditions $(47,48)$. Proteomic analysis of the hypothalamus revealed differences in neuropeptide levels between CONV-R and GF mice, and differential abundance of proteins related to the regulation of transmitter release, signaling pathways, and synapses (49). In addition, GF mice conventionalized with microbiotas from CONV-R mice exhibit upregulation of hypothalamic genes related to extracellular matrix (ECM) function compared with GF mice, suggesting that ECM modification may contribute to gut microbial effects on the hypothalamus (50).

While the precise mechanisms by which the gut microbiota alters hypothalamic physiology are poorly understood, direct actions of microbial metabolism and associated microbiota- 
Table 1. Gut microbiota-mediated changes in brain regions related to homeostatic feeding behavior

\begin{tabular}{|c|c|c|c|}
\hline Neurophysiological change & Conditions compared & Model organism & References \\
\hline \multicolumn{4}{|l|}{ Hypothalamus } \\
\hline Altered proteome (neuropeptides, transmitter release, signaling pathways, synapses) & CONV-R vs. GF & Mice & 49 \\
\hline Neurotransmitter synthesis (5-HT, dopamine, BDNF) affected by gut microbial aromatic AA metabolism & Ileal infusion of vehicle vs. antibiotics & Piglets & 51,52 \\
\hline SCFAs alter c-Fos expression, reduce food intake & Vehicle vs. SCFA administration & Mice & 55,57 \\
\hline ClpB stimulates POMC neurons, reduces food intake & Vehicle vs. E. coli protein injection & Rats & 60 \\
\hline Altered gene expression of GLP-1 and BDNF & CONV-R vs. GF & Mice & 47 \\
\hline LPS $\uparrow$ c-Fos expression & Vehicle vs. LPS injection & - & 64 \\
\hline Butyrate $\downarrow$ c-Fos expression & Vehicle vs. oral butyrate administration & Mice & 57 \\
\hline HFD induces microglial activation, vagal afferent reorganization & Low-fat diet vs. HFD & Rats & 65,66 \\
\hline \multicolumn{4}{|l|}{ Dorsal raphe nucleus } \\
\hline Altered $\Delta$ FosB activity & CONV-R vs. GF & Mice & 69 \\
\hline
\end{tabular}

derived molecules may be involved. In piglets, increased carbohydrate availability decreases gut microbial metabolism of aromatic amino acids (AAAs), which makes systemic AAAs more available for synthesis of neurotransmitters such as serotonin (5-HT), dopamine, and BDNF in the hypothalamus $(51,52)$. Microbial fermentation of nondigestible carbohydrates produces the shortchain fatty acids (SCFAs) acetate, propionate, and butyrate (53). SCFA administration reduces energy intake in rodent and human studies (54), potentially via direct effects on central neurons (acetate) or indirect signaling through peripheral circuits (propionate, butyrate) that innervate the hypothalamus (54-57). One key caveat regarding these studies is that SCFA administration leads to supraphysiological concentrations in the periphery, as the majority of endogenously produced SCFAs are metabolized by the colon and liver $(53,54)$. Intraperitoneal injection of acetate in mice reduces food intake and alters hypothalamic neuronal activity, gene expression, and neurotransmission (55). Dietary propionate supplementation induces the neuronal immediate-early activation marker c-Fos in hypothalamic nuclei, including the ARC and parabrachial nucleus, but, interestingly, does not appear to alter food intake in rats (56). Oral butyrate administration in mice reduces food intake, which requires intact vagal nerve signaling, and decreases c-Fos expression in hypothalamic NPY neurons (57). In addition to their neuronal effects, SCFAs also modulate glucose homeostasis $(53,56)$, and brain glucose sensing plays an important role in both homeostatic and hedonic feeding control $(58,59)$. Similarly to SCFAs, E. coli stationary-phase proteins acutely suppress food intake and induce c-Fos in hypothalamic POMC neurons in the ARC in rats (60). In particular, ClpB (bacterial mimetic of $\alpha-\mathrm{MSH}$, a cleaved product of POMC) stimulates the firing rate of hypothalamic POMC neurons (60). In summary, the gut microbiota regulates hypothalamic functions, and SCFAs and $\mathrm{ClpB}$ are promising candidate molecules that alter host feeding behavior via hypothalamic neurons. Further investigations into the neural pathways by which these molecules cause appetite suppression are warranted.

Brainstem. The brainstem processes energy-balance signals from the vagus nerve, circulating hormones, and metabolites, as well as descending signals from the midbrain and forebrain, and further relays the information to control motor, autonomic, and endocrine functions involved in feeding $(1,8,61)$. A key feeding-related brainstem nucleus is the nucleus of the solitary tract (NTS), which receives inputs from vagal gastrointestinal afferents and produces outputs to both homeostatic brain regions such as the ARC and dorsal motor nucleus of the vagus and higher-order regions such as the nucleus accumbens (NAc) $(8,61)$. Its connection to the vagus nerve provides a potential link for the gut microbiota to influence NTS function and downstream projection sites.

The gut microbiota has been reported to modulate neuropeptide expression, neuronal activity, and microglial activation in the NTS. CONV-R mice exhibit decreased expression of the anorexigenic neuropeptides glucagon-like peptide-1 (Glp-1) and $B d n f$ in the brainstem compared with GF mice (47). Since preproglucagon neurons in the NTS are the main source of GLP-1 in the brain (62, $63)$, this suggests that the gut microbiota modulates NTS function. Expression of c-Fos in the NTS can be induced by peripheral administration of the bacterial cell wall component lipopolysaccharide (LPS) (64) and decreased by oral butyrate administration (57), both of which are associated with reduced food intake. Highfat diet-induced (HFD-induced) shifts in the composition of the gut microbiota induce microglial activation $(65,66)$ and vagal afferent reorganization in the NTS, which are ameliorated by the antibiotic minocycline (65). Another brainstem nucleus that has recently been appreciated as regulating feeding is the dorsal raphe nucleus (DRN) $(67,68)$. GF mice show increased $\triangle$ FosB (a marker of long-term plasticity and cell activity) in the DRN compared with CONV-R mice, suggesting that the gut microbiota has effects on DRN activity (69). Whether such gut microbial modulation of the NTS and DRN actually leads to changes in feeding behavior remains to be seen and will need to be distinguished from effects on their downstream projection sites.

\section{Gut microbiota and non-homeostatic feeding controls}

Cortical regions. The prefrontal cortex (PFC), orbitofrontal cortex (OFC), and insula control motivational and rewarding aspects of 


\section{Table 2. Gut microbiota-mediated changes in brain regions related to non-homeostatic feeding behavior}

\begin{tabular}{|c|c|c|c|}
\hline Neurophysiological change & Conditions compared & Model organism & References \\
\hline \multicolumn{4}{|l|}{ Prefrontal cortex } \\
\hline $\begin{array}{l}\text { Altered transcriptome (synaptic LTP, steroid hormone metabolism, citrate cycle, } \\
\text { cAMP signaling) }\end{array}$ & CONV-R vs. GF & Mice & 86 \\
\hline Altered expression of BDNF, NGFI-A, and $\Delta$ FosB & CONV-R vs. GF & Mice & 86,87 \\
\hline $\begin{array}{l}\text { L. rhamnosus } \downarrow \text { GABA receptor A } \alpha 2 \text {, reduces anxiety- and depression-related } \\
\text { behavior }\end{array}$ & Vehicle vs. L. rhamnosus (JB-1) treatment & Mice & 88 \\
\hline Altered AA metabolism (D-serine) & CONV-R vs. GF & Mice & 92 \\
\hline $\begin{array}{l}\text { Prebiotic COS } \uparrow \text { NMDA receptor subunit } 1, \uparrow \text { cortical neuronal response to NMDA, } \\
\text { improve attention }\end{array}$ & Control vs. prebiotic Bimuno GOS (B-GOS) water & Rats & 94,95 \\
\hline \multicolumn{4}{|l|}{ Insula } \\
\hline Resting state functional connectivity correlates with gut microbiota diversity & - & Humans & 89 \\
\hline Functional connectivity associated with fecal indole levels & - & Humans & 113 \\
\hline Propionate reduces activity, reduces energy intake & Inulin vs. inulin-propionate ester & Humans & 114 \\
\hline \multicolumn{4}{|l|}{ Striatum } \\
\hline Altered dopamine turnover rate & CONV-R vs. GF & Mice, rats & 86,105 \\
\hline Altered AA metabolism & CONV-R vs. GF & Mice & 92 \\
\hline Bacterial phenylalanine synthesis correlates with striatal reward fMRI response & Control vs. ADHD cases & Humans & 106 \\
\hline Altered expression of dopamine receptors & Alcohol-seeking vs. resilient & Rats & 107 \\
\hline Altered synaptophysin and PSD-95 & CONV-R vs. GF & Mice & 86 \\
\hline Altered expression of peptidoglycan-sensing proteins during development & CONV-R vs. GF and antibiotics & Mice & 112 \\
\hline \multicolumn{4}{|l|}{ Ventral tegmental area } \\
\hline Prebiotic GOS and FOS $\uparrow$ BDNF, NMDA receptor subunits 1 and $2 A$ & Control vs. prebiotic FOS or GOS water & Mice & 94 \\
\hline L. rhamnosus $\uparrow$ GABA receptor $A \alpha 2$, glutamate, glutamine, $N$-acetyl aspartate & Vehicle vs. L. rhamnosus (JB-1) treatment & Mice & 88,130 \\
\hline Decreased memory scores & $\begin{array}{l}\text { Fecal microbiota transplant from obese vs. } \\
\text { nonobese humans }\end{array}$ & Mice & 136 \\
\hline A. muciniphila, probiotic VSL3 improve diet-induced cognitive deficits & $\begin{array}{l}\text { Vehicle vs. probiotic supplementation to HFD- } \\
\text { or cafeteria diet-fed mice }\end{array}$ & Mice & 137,138 \\
\hline
\end{tabular}

feeding (1). While the PFC classically inhibits impulsive feeding behavior (1,70-73), a subset of PFC neurons promotes food intake in sated animals $(74,75)$. The insula $(76,77)$ and OFC $(78,79)$ integrate sensory and internal state inputs to encode the perceived value of palatable foods that then influence feeding motivation (1). Cortical regions activated by hunger show similar patterns in rodents and humans (80), suggesting that findings from rodent studies of the cortex may apply to humans.

Recent studies of social and stress-related behaviors in rodents have implicated the gut microbiota in controlling development and function of the frontal cortex, particularly the PFC $(29,81)$. One of the mechanisms by which gut microbiota affect social behavior is regulating myelination in the $\mathrm{PFC}$, a region that plays a key role in planning and decision making $(29,81-85)$. Gut microbes regulate myelin gene expression and differentiation by producing metabolites such as $p$-cresol that could impair oligodendrocyte differentiation (82) and by affecting levels of microRNAs that target translation of myelin-related genes (85). In addition to myelin-related genes, comparison between GF and CONV-R mice reveals gut microbial regulation of genes involved in synaptic long-term potentiation (LTP), steroid hormone metabolism, citrate cycle, and cAMP-mediated signaling in the frontal cortex, hippocampus, and striatum (86). Supporting this regulation of synaptic changes, the gut microbiota also influences tran- 


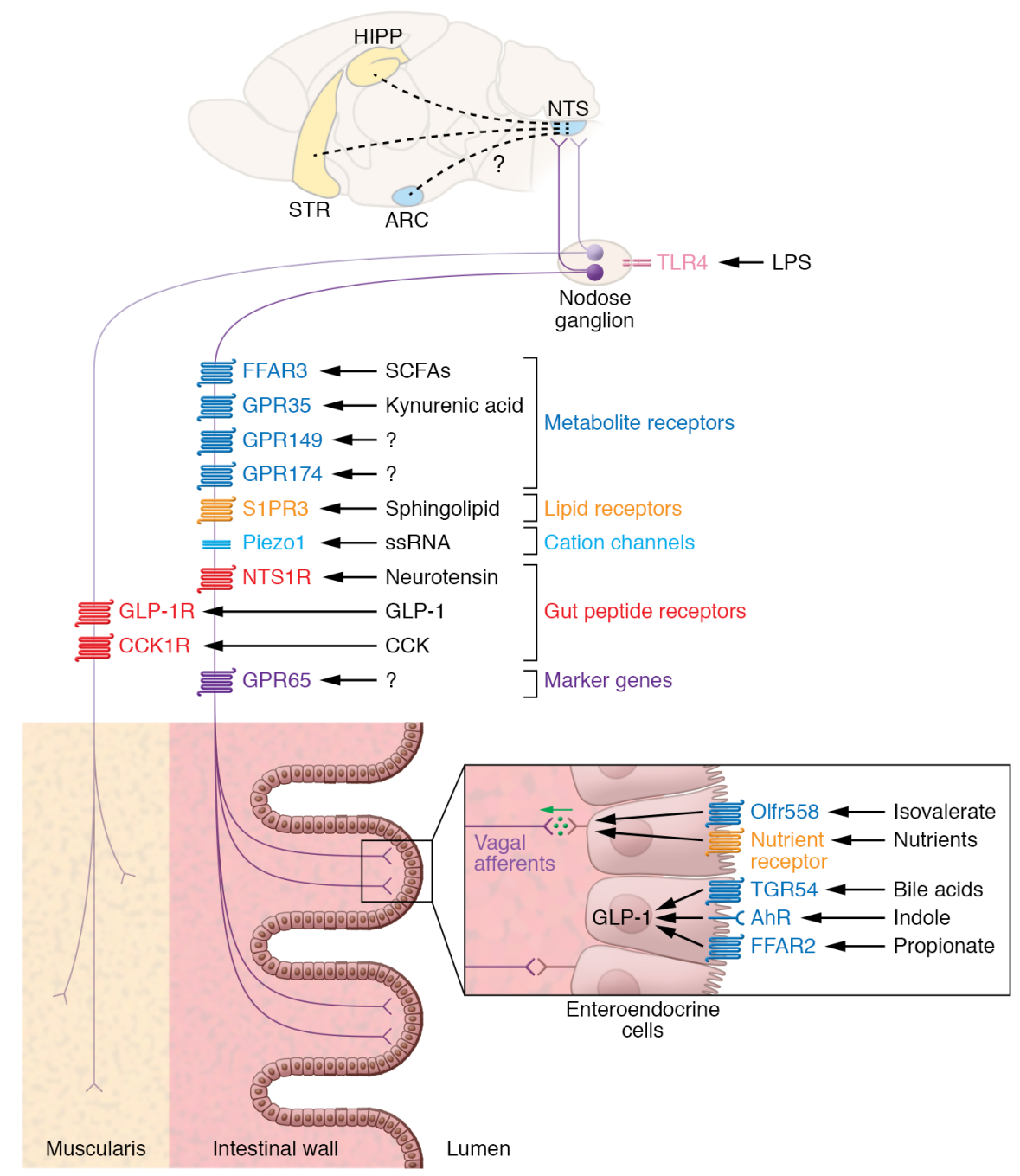

Figure 2. Proposed sites of action for microbial metabolites to influence vagal activity. Dotted lines indicate multisynaptic pathways. AhR, aryl hydrocarbon receptor; ARC, arcuate nucleus of the hypothalamus; CCK, cholecystokinin; CCK1R, CCK receptor 1; FFAR2, free fatty acid receptor 2 (formerly GPR43); FFAR3, free fatty acid receptor 3 (formerly GPR41); GLP-1, glucagon-like peptide-1; GLP-1R, GLP-1 receptor; HIPP, hippocampus; LPS, lipopolysaccharide; NTS, nucleus of the solitary tract; NTS1R, neurotensin receptor 1; Olfr558, olfactory receptor 558; S1PR3, sphingosine-1-phosphate receptor 3; STR, striatum; TCR5, Takeda G protein-coupled receptor 5; TLR4, Toll-like receptor 4. Adapted with permission from Molecular Metabolism (155).

script levels of synaptic plasticity genes and neurotransmitter receptors. GF mice show reduced gene expression of BDNF in the PFC (87) and nerve growth factor-inducible clone A (NGFI-A) in the PFC and OFC (86), and increased expression of $\triangle F o s B$ in the PFC (87), compared with CONV-R mice. Treatment of healthy CONV-R mice with Lactobacillus rhamnosus reduces gene expression of GABA receptor A 22 in the PFC and reduces anxiety- and depression-related behavior in a vagal nerve-dependent manner (88). The gut microbiota may also influence the insula, as is suggested by a correlation between insular resting state functional connectivity and gut microbial diversity in humans (89).

Overall, the gut microbiota can modulate myelination and synaptic gene expression in the frontal cortex with consequent effects on social and anxiety-related behaviors. Since cortical neural networks that control social and feeding behavior are closely intertwined (90), the gut microbiota may have the potential to influence feeding behavior through cortical regulation.

Although a causal link between gut microbiota and cortical regulation of feeding behavior remains to be established, a few studies suggest that one potential mechanism may involve gut microbial amino acid metabolism. Deficits in inhibitory control, which are mediated by the PFC, may contribute to unhealthy eating and exercise habits in obesity. Inhibitory control is associated with obesity-dependent alterations in gut bacterial one-carbon and aromatic amino acid metabolic pathways in human cohorts (91). Furthermore, fecal microbiota transplant from obese individuals is sufficient to alter reversal learning and medial PFC gene expression in recipient mice (91). This study suggests that the gut microbiota may modulate a cognitive function involved in feeding via the PFC. Another study shows that gut microbes regulate amino acid metabolism in various brain regions, including decreased cortical D-serine in CONV-R compared with GF mice (92). D-serine acts as a coagonist with glutamate on NMDA receptors (93). Prebiotic supplementation with galacto-oligosaccharides (GOS), which induces gut microbiota proliferation, increases protein expression of NMDA subunit 1 and levels of $\mathrm{D}$-serine in the frontal cortex, enhances cortical neuronal responses to NMDA, and improves cognitive performance in rats $(94,95)$. Because NMDA receptors and D-serine have been implicated in control of appetite, food preference, and reinforcement learning $(93,96,97)$, gut microbial influence on this system in the frontal cortex could potentially alter host feeding behavior. Future studies investigating the effects of altering microbial amino acid metabolism in the PFC via microbiota transplant or prebiotics on host food intake and preference are necessary.

Mesolimbic dopamine pathway. The mesolimbic dopamine pathway, from the ventral tegmental area (VTA) to the NAc locat- 
ed in the ventral striatum, encodes food-associated reward, which can override homeostatic signals to promote excessive consumption $(1,6,70,98-100)$. Both the VTA and the NAc are modulated by homeostatic information from the hypothalamus $(98,101)$ and gut hormones $(1,102)$. Growing evidence suggests that the gut microbiota also regulates the mesolimbic pathway by modifying dopaminergic transmission with potential effects on reward-associated behavior $(103,104)$. One study showed that GF rats have a lower dopamine turnover rate in the striatum than CONV-R rats (105), while another study reported that GF mice have higher dopamine, $5-\mathrm{HT}$, and noradrenaline turnover rates in the striatum than CONV-R mice (86). This difference may be explained by differences in rodent species and strain, but they nevertheless implicate a role for gut microbiota in dopamine metabolism. One potential mechanism by which the gut microbiota affects neurotransmitter levels is through the modification of amino acid metabolism (92, 106). Predicted increases in a bacterial enzyme for the synthesis of the dopamine precursor phenylalanine correlate with decreased ventral striatal functional MRI (fMRI) responses during reward anticipation in humans, suggesting a link between gut microbial metabolism and behavior (106).

In addition to dopamine itself, the gut microbiota also affects dopamine receptor expression and other synaptic proteins. GF mice show significantly higher protein expression of synaptophysin and PSD-95 than CONV-R mice (86). Gut microbiota composition also correlates with increases in striatal gene expression of dopamine D1 receptor, decreases in striatal expression of dopamine D2 receptor, and changes in impulsivity measures in a rat model of alcohol seeking (107).

The gut microbiota can also influence host behavior through mesolimbic structures. Treatment of maternal HFD offspring or autism mouse models with Lactobacillus reuteri corrects social deficits by rescuing social interaction-induced synaptic plasticity in the VTA in a vagal nerve-dependent manner $(108,109)$. Changes in gut microbiota are associated with anxiety- and depression-like behavior in mice with diet-induced obesity through decreased insulin signaling and increased inflammation in the NAc and amygdala, which can be transferred to GF mice by fecal transplant and improved by antibiotic treatment (110). Relevant to feeding behavior, prebiotic supplementation with fructo-oligosaccharides (FOS), which alters gut microbiota composition, can modify motivation to eat a high-fat, high-sugar (HFHS) diet in mice. When mice are fed FOS after chronic HFHS exposure, they show decreased palatable food tropism and consumption, associated with normalization of NAc expression of genes involved in dopamine signaling (111).

Despite these associations between gut microbiota, mesolimbic physiological changes, and behavior, the underlying mechanisms of gut microbial regulation of the mesolimbic pathway remain unclear. However, some studies have suggested that bacterial molecules can act directly or indirectly on the striatum. In mice, the developing striatum expresses proteins that detect the bacterial cell wall component peptidoglycan, and their expression is sensitive to gut microbiota depletion, suggesting that the gut microbiota modifies striatal development via peptidoglycan (112). In humans, indole metabolites and propionate may alter mesolimbic activity through indirect gastrointestinal or vagal pathways $(113,114)$. Fecal levels of indole metabolites strongly associate with functional connectivity of the NAc, potentially interacting with GLP-1-producing enteroendocrine L cells or serotonergic enterochromaffin cells to act on vagal afferents that then communicate with central reward regions (113). Increasing colonic propionate levels in humans reduce activity in the NAc and caudate nucleus, which correlates with a decrease in subjective appeal of high-energy food pictures and in energy intake during an ad libitum meal (114). Thus, studies in both rodents and humans suggest a relationship between the gut microbiota and mesolimbic dopamine pathway in modulating feeding behavior $(111,114)$ through gene expression changes and metabolites such as propionate and indoles. Future studies are needed to elucidate the neural pathways by which these metabolites affect feeding behavior and to identify candidate metabolites that can impact the VTA in addition to the NAc.

Hippocampus. The hippocampus regulates food intake by integrating information about the external visuospatial environment, meal-related memories, learned experiences, and energy status $(1,115,116)$. The ventral subregion (vHP) helps consolidate feeding-related learned associations by sensing endocrine signals and projecting to higher brain regions including the PFC and NAc (1, 117). Higher body mass index correlates with reduced hippocampal volume, suggesting that hippocampal dysregulation may contribute to obesity (118-120). The gut microbiota affects many aspects of hippocampal physiology, including structure, neurogenesis, gene expression, and neurochemistry (29). In mice, the gut microbiota is required to maintain hippocampal structure, with GF mice showing increased CA2/3 volume and dendritic atrophy of vHP pyramidal neurons compared with CONV-R mice (121). Supporting this notion in humans, increases in taxonomic diversity of the gut microbiota correlate with sparing of hippocampal microstructure (46). The gut microbiota (29) and dietary changes (122) also affect hippocampal neurogenesis, which is important for maintenance of spatial memory and learning capacity (122). One study found that broad-spectrum antibiotic treatment of CONV-R mice decreases neurogenesis in the subgranular zone of the dentate gyrus, which could be ameliorated by postnatal treatment with the probiotic VSL3, which comprises a mixture of several commensal bacterial species (123). Another study found that GF mice show increased neurogenesis in the dorsal hippocampus compared with CONV-R mice, which could not be corrected by postnatal conventionalization (124). This difference may be explained by physiological differences between GF rearing and postnatal antibiotic treatment (29). Furthermore, the gut microbiota affect microglia, which are important for brain development including synaptic pruning and remodeling throughout adulthood (125). GF mice show defective microglial maturation and decreased reactivity in the hippocampus compared with CONV-R or conventionalized GF mice (125, 126), associated with abnormally increased synaptic density and decreased neuronal firing rate (126). Microglial abnormalities are corrected by recolonization with complex microbiota $(125,126)$, SCFAs (125), and a limited consortium of Bifidobacterium species (126), suggesting that specific microbes and metabolites can regulate microglial homeostasis and, consequently, synaptic function.

In addition to altering synaptic function, the gut microbiota influences expression of synapse-related genes in the hippocam- 
pus (29). Conventionalized GF mice show downregulation of genes involved in LTP compared with GF mice (126). GF (86) and antibiotic-treated mice (127) also exhibit decreased hippocampal expression of BDNF and its receptor TrkB (128) compared with CONV-R mice. Gut microbiota may also impact neurotransmitter levels: GF mice display increased levels of hippocampal 5-HT and its metabolite 5-hydroxyindoleacetic acid (129) and decreased levels of GABA and L-glutamine (92) in the hippocampus. Prebiotic and probiotic treatments alter levels of neurotransmitters and their receptors, supporting their regulation by gut microbes. Prebiotic treatment with FOS and GOS increases hippocampal gene and protein expression of BDNF and NMDA receptor subunits 1 and 2A (94). Treatment of healthy CONV-R mice with L. rhamnosus increases gene expression of GABA receptor $\mathrm{A} \alpha 2$ in the hippocampus (88) and increases hippocampal glutamate, glutamine, and $N$-acetyl aspartate levels (130).

These neurophysiological changes may contribute to influences of the gut on hippocampus-dependent learning and memory $(29,131)$. While causal effects of the gut microbiota on hippocampal feeding regulation remain to be proven, the assays used in rodent models of microbiota depletion or probiotic treatment - novel object recognition, Barnes maze, and Morris water maze suggest it is possible. Antibiotic (29) and probiotic treatments (29, 131) modulate spatial memory in mice, as measured in the Barnes maze and Morris water maze (132). Spatial memory is an important component of food-place memory, which is encoded by hippocampal dopamine 2 receptor (hD2R) neurons that regulate food intake (116). GF, antibiotic-treated, and probiotic-treated mice also show altered behavior in the novel object recognition test, which tests working memory but may also hint at contextual memory (133). Episodic memory, which involves "what-where-when" experiences, significantly affects food intake in human studies (115) and can be evaluated in mice using object-place-context tests $(134,135)$. Future studies are needed to establish a causal connection between gut microbiota and hippocampal feeding-related functions, including tests for food-specific episodic $(134,135)$ and food-place (116) memory in microbiota-depleted or probiotic-treated mice. Profiling hD2R neurons (116) in microbiota-depleted compared with CONV-R mice would also be of interest.

Supporting this potential role of the gut microbiota in regulating feeding-related memory, a few recent studies have reported links between gut microbes and diet-induced and obesity-associated memory deficits (136-138). Gut microbiota composition and plasma and fecallevels of AAAs are associated with short-term and working memory in humans with obesity (136). Fecal microbiota transplantation from obese individuals leads to decreased memory scores in recipient mice in comparison with nonobese donors (136). In another study, early-life HFD impairs hippocampusdependent contextual and spatial learning and memory in mice by altering the gut microbiota, specifically by depleting Akkermansia muciniphila (137). Fecal transplantation and LPS treatment are sufficient to confer these memory deficits to chow-fed mice, suggesting a causal role of the gut microbiota (137). Furthermore, treatment of HFD-fed mice with A. muciniphila ameliorates these memory deficits along with hippocampal neuronal development and LTP (137). HFD-induced memory deficits and hippocampal gene expression changes can also be prevented by probiotic treatment with VSL3 in rats (138). Together, these studies indicate the potential for specific microbes to affect hippocampal function and memory in obesity, in which cognitive deficits could predispose individuals to overeating (136). Future studies are needed to elucidate the neural mechanisms underlying gut microbial influences on hippocampal function in obesity, which may have therapeutic implications.

\section{Gut microbiota and vagal nerve}

The vagus nerve controls food intake by relaying chemosensory and mechanosensory information from the gastrointestinal tract to the brain to convey feeding-related signals $(7-10,139)$. Vagal afferent neurons expressing GPR65 extend to intestinal villi and respond to intestinal nutrients in mice (140). A subset of enteroendocrine cells directly sense nutrient and other chemical stimuli, and quickly transduce these gut lumenal signals to vagal neurons through glutamatergic and serotonergic synapses (141, 142). Mechanosensing vagal afferents expressing GLP-1 receptor extend axons to the muscle layers to sense gastrointestinal stretch $(140,143)$. Interestingly, a recent study found that activation of mechanosensing vagal neurons, rather than nutrient-sensing ones, suppresses food intake through inhibition of hypothalamic AgRP neurons (143) and through parabrachial prodynorphin neurons (144); however, the contribution of chemosensation to feeding control has not been excluded (8). Moreover, the effect of GPR $65^{+}$vagal afferents on behavior remains unknown (139). In addition to chemosensory and mechanosensory information, intact vagal signaling is required to facilitate actions of hormonal satiety signals, particularly cholecystokinin (CCK), on feeding behavior $(7,9,139)$. Although it classically participates in negative feedback control of food intake (9), recent studies have revealed new roles for the vagus nerve in stimulating feeding and learning nutrient preferences through neural pathways involving the NTS and downstream projection sites.

Vagal afferent neurons first terminate in the NTS, located in the brainstem (7). NTS neurons coexpressing NPY and epinephrine that receive vagal afferents stimulate feeding, showing an orexigenic role for the vagus nerve (145). The vagus nerve also forms multisynaptic pathways via the NTS to higher-order brain regions (61) involved in non-homeostatic feeding controls, such as the striatum $(146,147)$ and hippocampus $(148,149)$, and mediates brain functions in mice. Vagal activation induces dopamine release from the substantia nigra and conditions flavor and place preference (146). Vagal neurons, specifically those expressing CCK receptors, are also necessary for hippocampus-dependent episodic and spatial memory (148) and influence hippocampal neurogenesis $(148,149)$. Consistent with its effects on reward and memory, the vagus nerve has recently been appreciated as modulating nutrient preference learning $(10,150)$. Vagal nerve signaling is key to developing preference for sugar over artificial sweetener independent of taste in mice $(151,152)$ through activation of caudal NTS (151) and dopaminergic VTA neurons (152). Both vagal innervation and duodenal sensing are also required for preference learning for fats and proteins in mice (153). Although all these studies were conducted in rodents, evidence from gastric bypass surgeries and nerve stimulation suggests that the vagus nerve may also play a role in food intake control in humans (150). 
Overall, the vagus nerve plays essential roles in both homeostatic and non-homeostatic feeding behavior by modulating appetite and nutrient preference learning. Owing to its proximity to the gut microbiota in the gastrointestinal lumen, it serves as an appealing potential pathway by which gut microbes may affect host feeding behavior that warrants further investigation. Vagal neurons express numerous G protein-coupled receptors (GPCRs) $(154,155)$, and can be subclassified based on their expression of receptorencoding genes Gpr65, Htr3a/b, Piezo1, Ntsr1, Cysltr2, Gpr174, and S1pr3 (154). Intestinal GPR65+ vagal neurons also express Gpr35, Cbr1 (encoding carbonyl reductase 1), Gpr149, Gpr161, and Crh2 (encoding corticotropin-releasing hormone 2) (155). Gut microbial metabolites can interact with some of these GPCRs based on evidence from high-throughput screening of microbial ligand-receptor binding $(156,157)$. Of the aforementioned receptors, metabolomes modulated by the human gut microbiota are predicted to affect Ntsr1, S1pr3, Gpr35, Gpr149, and Gpr174 (156). GPR35 binds aromatic, acidic metabolites such as the tryptophan metabolite kynurenic acid (155), which is modulated by gut microbes (158). S1PR3 binds sphingolipids, which are also produced and modulated by gut microbes (159). PIEZO1 channels have also been reported to bind fecal RNA potentially derived from gut microbes (160). In addition to direct activation of receptors on vagal neurons, gut luminal microbial metabolites such as butyrate, isobutyrate, and isovalerate may also bind receptors on enterochromaffin cells, stimulating the release of secondary metabolites that activate vagal afferents (142).

Some mechanisms by which microbial metabolites can interact with enteroendocrine cells and vagal neurons to alter feeding behavior have been reported to involve SCFAs, bile acids, and indoles $(20,161)$. SCFAs can act directly on vagal neurons, which express the SCFA receptor FFAR3 (also called GPR41) $(156,162)$, to control food intake. Intraperitoneal injection of SCFAs, especially butyrate, reduces food intake in fasted mice, which is attenuated by hepatic vagotomy and by capsaicin-induced sensory denervation (163). Furthermore, butyrate injection induces cellular activation markers in the nodose ganglion and medial NTS and can activate intracellular calcium signaling in vagal neurons ex vivo (163). SCFAs can also affect nutrient sensing and hormone production by enteroendocrine cells to indirectly affect vagal signaling. Treatment of enteroendocrine cell cultures with propionate and butyrate increases mRNA levels of umami taste receptors, suggesting that SCFAs can alter enteroendocrine sensitivity to nutrients (164). Propionate can also induce GLP-1 secretion by enteroendocrine L cells and colonic crypts via the receptor FFAR2 (also called GPR43) (165-167). Similarly, indole (168) and bile acids $(162,169)$ can also induce GLP-1 production by enteroendocrine cells, which then stimulates colonic vagal afferents (168).

Aside from metabolites, vagal neurons may interact with bacterial components, namely LPS via TLR4 receptors. Chronic LPS treatment leads to reduced satiation and hyperphagia in rats by inhibiting vagal leptin signaling that results in CCK resistance (170-173). HFD-driven gut microbiota dysbiosis and inflammation, including increased serum LPS levels, are also associated with vagal remodeling - withdrawal of vagal afferents from the gut and NTS and microglial activation at the nodose ganglion - which is ameliorated by antibiotic treatment $(65,170,174)$. These findings suggest that endotoxemia can contribute to the development of obesity via the vagus nerve. In summary, the vagus nerve serves as a likely pathway for gut microbes to modulate host feeding behavior through direct receptor activation or indirect interactions with enteroendocrine cells (Figure 2). Future studies are needed to better understand mechanisms by which the gut microbiota interacts with the vagus nerve, particularly identifying microbial ligands for orphan GPCRs and discovering the downstream effects of activating those receptors. Studies testing effects of vagal receptor activation by microbial ligands on feeding behavior are also needed in addition to current vagal ablation strategies. This understanding could contribute to developing microbiota-based vagal modulation strategies to treat obesity and eating disorders, which may refine and extend existing electrical interventions (175).

\section{Conclusion}

An increasing number of studies together suggest that the gut microbiota affects the structure and function of neural pathways that play important roles in regulating both homeostatic and non-homeostatic feeding behaviors. However, these studies largely use bulk depletion of the microbiota in animal models to provide proof-of-concept that an intact microbiota is required to maintain an aspect of gene expression, biochemical profile, or neurophysiology. More studies are needed to determine which of these findings are reproducible across different animal models and microbiota paradigms, and to provide more rigorous evidence that the microbiota affects feeding-related neural circuits. Further, integrative studies are necessary to determine whether observed changes in relevant neural circuits actually render downstream alterations in host feeding behavior. If so, detailed understanding of specific microbial functions and signaling pathways across the gut-brain axis would be needed to dissect underlying signaling mechanisms. There remains a knowledge gap in microbial effects on specific subtypes of brain neurons compared with neurons of the vagus nerve. Recent advances in single-cell sequencing (176) may serve as a launching point for future studies on gut microbiota and neuronal subtypes in obesity. Corresponding human studies that manipulate specific microbes and metabolites will be important for assessing host-microbe interactions that occur in humans and their potential relevance to metabolic and eating disorders. Understanding the influence of the gut microbiota on homeostatic and non-homeostatic feeding behaviors may reveal novel insights into the biological underpinnings of food choice, with the potential to inform new approaches for modifying food preferences in obesity and eating disorders (177).

\section{Acknowledgments}

KBY is supported by funds from the UCLA Whitcome Fellowship. EYH is supported by funds from the Army Research Office Multidisciplinary University Research Initiative (W911NF-17-0402) and Chan Zuckerberg Initiative (2018-191860); is a New York Stem Cell Foundation Robertson Investigator; and is supported in support by the New York Stem Cell Foundation.

Address correspondence to: Kristie B. Yu or Elaine Y. Hsiao, Department of Integrative Biology \& Physiology, 610 Charles E. Young Drive East, University of California, Los Angeles, California 90095, USA. Email: kristie.yu15@gmail.com (KBY). Email: ehsiao@g.ucla.edu (EYH). 
1. Liu CM, Kanoski SE. Homeostatic and nonhomeostatic controls of feeding behavior: distinct vs. common neural systems. Physiol Behav. 2018;193(pt B):223-231.

2. Morton GJ, et al. Neurobiology of food intake in health and disease. Nat Rev Neurosci. 2014;15(6):367-378.

3. Clifton PG. Neural circuits of eating behaviour: Opportunities for therapeutic development. JPsychopharmacol. 2017;31(11):1388-1402.

4. Sternson SM, et al. Neural circuits and motivational processes for hunger. Curr Opin Neurobiol. 2013;23(3):353-360.

5. Kenny PJ. Reward mechanisms in obesity: new insights and future directions. Neuron. 2011;69(4):664-679.

6. Gupta A, et al. Brain-gut-microbiome interactions in obesity and food addiction. Nat Rev Gastroenterol Hepatol. 2020;17(11):655-672.

7. Waise TMZ, et al. The metabolic role of vagal afferent innervation. Nat Rev Gastroenterol Hepatol. 2018;15(10):625-636.

8. Moura-Assis A, et al. Gut-to-brain signals in feeding control. Am J Physiol Metab. 2021;320(2):E326-E332.

9. Schwartz GJ. Roles for gut vagal sensory signals in determining energy availability and energy expenditure. Brain Res. 2018;1693(pt B):151-153.

10. Shechter A, Schwartz GJ. Gut-brain nutrient sensing in food reward. Appetite. 2018;122:32-35.

11. Fetissov SO. Role of the gut microbiota in host appetite control: bacterial growth to animal feeding behaviour. Nat Rev Endocrinol. 2017;13(1):11-25

12. Alcock J, et al. Is eating behavior manipulated by the gastrointestinal microbiota? Evolutionary pressures and potential mechanisms. Bioessays. 2014;36(10):940-949.

13. Turnbaugh PJ, et al. An obesity-associated gut microbiome with increased capacity for energy harvest. Nature. 2006;444(7122):1027-1031.

14. Ridaura VK, et al. Gut microbiota from twins discordant for obesity modulate metabolism in mice. Science. 2013;341(6150):1241214.

15. Nicholson JK, et al. Host-gut microbiota metabolic interactions. Science. 2012;336(6086):1262-1267.

16. Sonnenburg JL, Bäckhed F. Diet-microbiota interactions as moderators of human metabolism. Nature. 2016;535(7610):56-64.

17. Schéle $\mathrm{E}$, et al. Regulation of body fat mass by the gut microbiota: possible mediation by the brain. Peptides. 2016;77:54-59.

18. Heiss CN, Olofsson LE. Gut microbiota-dependent modulation of energy metabolism. J Innate Immun. 2018;10(3):163-171.

19. Lindsay EC, et al. The potential role of the gut microbiota in shaping host energetics and metabolic rate. J Anim Ecol. 2020;89(11):2415-2426.

20. van de Wouw $M$, et al. Microbiota-gut-brain axis: modulator of host metabolism and appetite. J Nutr. 2017;147(5):727-745.

21. Chen $Z$, et al. Incorporation of therapeutically modified bacteria into gut microbiota inhibits obesity. J Clin Invest. 2014;124(8):3391-3406.

22. Lee P, et al. Gut microbiota and obesity: an opportunity to alter obesity through faecal microbiota transplant (FMT). Diabetes Obes Metab.
2019;21(3):479-490.

23. Agus A, et al. Gut microbiota-derived metabolites as central regulators in metabolic disorders. Gut. 2021;70(6):1174-1182.

24. Glenny EM, et al. Eating disorders and the intestinal microbiota: mechanisms of energy homeostasis and behavioral influence. Curr Psychiatry Rep. 2017;19(8):51.

25. Ruusunen $\mathrm{A}$, et al. The gut microbiome in anorexia nervosa: relevance for nutritional rehabilitation. Psychopharmacology (Berl). 2019;236(5):1545-1558.

26. Fetissov SO, Hökfelt T. On the origin of eating disorders: altered signaling between gut microbiota, adaptive immunity and the brain melanocortin system regulating feeding behavior. Curr Opin Pharmacol. 2019;48:82-91.

27. Roubalová $R$, et al. Anorexia nervosa: gut microbiota-immune-brain interactions. Clin Nutr. 2020;39(3):676-684.

28. Sampson TR, Mazmanian SK. Control of brain development, function, and behavior by the microbiome. Cell Host Microbe. 2015;17(5):565-576.

29. Vuong HE, et al. The microbiome and host behavior. Annu Rev Neurosci. 2017;40(1):21-49.

30. Cryan JF, et al. The microbiota-gut-brain axis. Physiol Rev. 2019;99(4):1877-2013.

31. O'Donnell MP, et al. A neurotransmitter produced by gut bacteria modulates host sensory behaviour. Nature. 2020;583(7816):415-420.

32. Henriques SF, et al. Metabolic cross-feeding in imbalanced diets allows gut microbes to improve reproduction and alter host behaviour. Nat Commun. 2020;11(1):1-15.

33. Leitão-Gonçalves R, et al. Commensal bacteria and essential amino acids control food choice behavior and reproduction. PLoS Biol. 2017;15(4):1-29.

34. Turner A, et al. Interactions between bitter taste, diet and dysbiosis: consequences for appetite and obesity. Nutrients. 2018;10(10):1336.

35. Swartz TD, et al. Up-regulation of intestinal type 1 taste receptor 3 and sodium glucose luminal transporter-1 expression and increased sucrose intake in mice lacking gut microbiota. $\mathrm{Br} J$ Nutr. 2012;107(5):621-630

36. Moran AW, et al. Nutrient sensing of gut luminal environment. Proc Nutr Soc. 2021;80(1):29-36.

37. Bernard A, et al. A preventive prebiotic supplementation improves the sweet taste perception in diet-induced obese mice. Nutrients. 2019;11(3):E549.

38. Cani PD, et al. Endocannabinoids-at the crossroads between the gut microbiota and host metabolism. Nat Rev Endocrinol. 2016;12(3):133-143.

39. Castonguay-Paradis S, et al. Dietary fatty acid intake and gut microbiota determine circulating endocannabinoidome signaling beyond the effect of body fat. Sci Rep. 2020;10(1):1-11.

40. Manca C, et al. Alterations of brain endocannabinoidome signaling in germ-free mice. Biochim Biophys Acta Mol Cell Biol Lipids. 2020;1865(12):158786.

41. Avalos $\mathrm{B}$, et al. Cannabinoid $\mathrm{CB}_{1}$ receptors in the intestinal epithelium are required for acute western-diet preferences in mice. Nutrients. 2020;12(9):2874.
42. Sohn JW. Network of hypothalamic neurons that control appetite. BMB Rep. 2015;48(4):229-233.

43. Wright $\mathrm{H}$, et al. Differential effects of hunger and satiety on insular cortex and hypothalamic functional connectivity. Eur J Neurosci. 2016;43(9):1181-1189.

44. Luo S, et al. Resting state hypothalamic response to glucose predicts glucose-induced attenuation in the ventral striatal response to food cues. Appetite. 2017;116:464-470.

45. Lizarbe B, et al. Imaging hypothalamic activity using diffusion weighted magnetic resonance imaging in the mouse and human brain. Neuroimage. 2013;64(1):448-457.

46. Fernandez-Real JM, et al. Gut microbiota interacts with brain microstructure and function. J Clin Endocrinol Metab. 2015;100(12):4505-4513.

47. Schéle E, et al. The gut microbiota reduces leptin sensitivity and the expression of the obesitysuppressing neuropeptides proglucagon $(\mathrm{Gcg})$ and brain-derived neurotrophic factor (Bdnf) in the central nervous system. Endocrinology. 2013;154(10):3643-3651.

48. Yao H, et al. Effects of gut microbiota on leptin expression and body weight are lessened by highfat diet in mice. Br J Nutr. 2020;124(4):396-406.

49. Li L, et al. Integrated label-free and 10-plex DiLeu isobaric tag quantitative methods for profiling changes in the mouse hypothalamic neuropeptidome and proteome: assessment of the impact of the gut microbiome. Anal Chem. 2020;92(20):14021-14030.

50. Qi X, et al. Extracellular matrix and oxidative phosphorylation: important role in the regulation of hypothalamic function by gut microbiota. Front Genet. 2020;11:520.

51. Gao K, et al. Antibiotics-induced modulation of large intestinal microbiota altered aromatic amino acid profile and expression of neurotransmitters in the hypothalamus of piglets. JNeurochem. 2018;146(3):219-234.

52. Gao K, et al. Increasing carbohydrate availability in the hindgut promotes hypothalamic neurotransmitter synthesis: aromatic amino acids linking the microbiota-brain axis. J Neurochem. 2019;149(5):641-659.

53. Koh A, et al. From dietary fiber to host physiology: short-chain fatty acids as key bacterial metabolites. Cell. 2016;165(6):1332-1345.

54. Blaak EE, et al. Short chain fatty acids in human gut and metabolic health. Benef Microbes. 2020;11(5):411-455.

55. Frost $\mathrm{G}$, et al. The short-chain fatty acid acetate reduces appetite via a central homeostatic mechanism. Nat Commun. 2014;5:3611.

56. De Vadder F, et al. Microbiota-generated metabolites promote metabolic benefits via gut-brain neural circuits. Cell. 2014;156(1-2):84-96.

57. Li Z, et al. Butyrate reduces appetite and activates brown adipose tissue via the gut-brain neural circuit. Gut. 2018;67(7):1269-1279.

58. Novin D, et al. Is there a role for the liver in the control of food intake? Am JClin Nutr. 1985;42(5 suppl):1050-1062.

59. Steinbusch L, et al. Brain glucose sensing in homeostatic and hedonic regulation. Trends Endocrinol Metab. 2015;26(9):455-466.

60. Breton J, et al. Gut commensal E. coli proteins 
activate host satiety pathways following nutrientinduced bacterial growth. Cell Metab. 2016;23(2):324-334.

61. Grill HJ, Hayes MR. Hindbrain neurons as an essential hub in the neuroanatomically distributed control of energy balance. Cell Metab. 2012;16(3):296-309.

62. Holt MK, et al. Preproglucagon neurons in the nucleus of the solitary tract are the main source of brain GLP-1, mediate stress-induced hypophagia, and limit unusually large intakes of food. Diabetes. 2019;68(1):21-33.

63. Diz-Chaves Y, et al. Glucagon-like peptide-1 (GLP-1) in the integration of neural and endocrine responses to stress. Nutrients. 2020;12(11):3304.

64. Chaskiel L, et al. Brainstem metabotropic glutamate receptors reduce food intake and activate dorsal pontine and medullar structures after peripheral bacterial lipopolysaccharide administration. Neuropharmacology. 2016;107:146-159.

65. Vaughn AC, et al. Energy-dense diet triggers changes in gut microbiota, reorganization of gutbrain vagal communication and increases body fat accumulation. Acta Neurobiol Exp (Wars). 2017;77(1):18-30.

66. Minaya DM, et al. Consumption of a high energy density diet triggers microbiota dysbiosis, hepatic lipidosis, and microglia activation in the nucleus of the solitary tract in rats. Nutr Diabetes. 2020;10(1):20.

67. Nectow AR, et al. Identification of a brainstem circuit controlling feeding. Cell. 2017;170(3):429-442.

68. Bruschetta $\mathrm{G}$, et al. $\mathrm{MC}_{4} \mathrm{R}$ signaling in dorsal raphe nucleus controls feeding, anxiety, and depression. Cell Rep. 2020;33(2):108267.

69. Campos AC, et al. Absence of gut microbiota influences lipopolysaccharide-induced behavioral changes in mice. Behav Brain Res. 2016;312:186-194.

70. He Q, et al. Poor ability to resist tempting calorie rich food is linked to altered balance between neural systems involved in urge and self-control. Nutr J. 2014;13:92.

71. Wood SMW, et al. Emotional eating and routine restraint scores are associated with activity in brain regions involved in urge and self-control. Physiol Behav. 2016;165:405-412.

72. Warthen DM, et al. Activation of pyramidal neurons in mouse medial prefrontal cortex enhances food-seeking behavior while reducing impulsivity in the absence of an effect on food intake. Front Behav Neurosci. 2016;10:63.

73. Anastasio NC, et al. Convergent neural connectivity in motor impulsivity and high-fat food bingelike eating in male Sprague-Dawley rats. Neuropsychopharmacology. 2019;44(10):1752-1761.

74. Land BB, et al. Medial prefrontal D1 dopamine neurons control food intake. Nat Neurosci. 2014;17(2):248-253.

75. Petrovich GD, et al. Medial prefrontal cortex is necessary for an appetitive contextual conditioned stimulus to promote eating in sated rats. JNeurosci. 2007;27(24):6436-6441.

76. Wu Y, et al. The anterior insular cortex unilaterally controls feeding in response to aversive visceral stimuli in mice. Nat Commun. 2020;11(1):640.
77. Avery JA, et al. Convergent gustatory and viscerosensory processing in the human dorsal midinsula. Hum Brain Mapp. 2017;38(4):2150-2164.

78. Seabrook LT, Borgland SL. The orbitofrontal cortex, food intake and obesity. J Psychiatry Neurosci. 2020;45(5):304-312.

79. Suzuki S, et al. Elucidating the underlying components of food valuation in the human orbitofrontal cortex. Nat Neurosci. 2017;20(12):1780-1786.

80. Benítez A, et al. Cerebral hunger maps in rodents and humans by diffusion weighted MRI. Appetite. 2019;142:104333.

81. Ntranos A, Casaccia P. The microbiome-gutbehavior axis: crosstalk between the gut microbiome and oligodendrocytes modulates behavioral responses. Neurotherapeutics. 2018;15(1):31-35.

82. Gacias M, et al. Microbiota-driven transcriptional changes in prefrontal cortex override genetic differences in social behavior. Elife. 2016;5:e13442.

83. Hoban AE, et al. Regulation of prefrontal cortex myelination by the microbiota. Transl Psychiatry. 2016;6:e774.

84. Keogh CE, et al. Myelin as a regulator of development of the microbiota-gut-brain axis. Brain Behav Immun. 2021;91:437-450.

85. Hoban AE, et al. Microbial regulation of microRNA expression in the amygdala and prefrontal cortex. Microbiome. 2017;5(1):102.

86. Heijtz RD, et al. Normal gut microbiota modulates brain development and behavior. Proc Natl Acad Sci U S A. 2011;108(7):3047-3052.

87. Kamimura I, et al. Microbial colonization history modulates anxiety-like and complex social behavior in mice [published online February 1 , 2020]. Neurosci Res. https://doi.org/10.1016/j. neures.2020.01.001.

88. Bravo JA, et al. Ingestion of Lactobacillus strain regulates emotional behavior and central GABA receptor expression in a mouse via the vagus nerve. Proc Natl Acad Sci U S A. 2011;108(38):16050-16055

89. Curtis K, et al. Insular resting state functional connectivity is associated with gut microbiota diversity. Eur J Neurosci. 2019;50(3):2446-2452.

90 . Jennings $\mathrm{JH}$, et al. Interacting neural ensembles in orbitofrontal cortex for social and feeding behaviour. Nature. 2019;565(7741):645-649

91. Arnoriaga-Rodríguez M, et al. Obesity-associated deficits in inhibitory control are phenocopied to mice through gut microbiota changes in one-carbon and aromatic amino acids metabolic pathways [published online January 29, 2021]. Gut. https://doi.org/10.1136/gutjnl-2020-323371.

92. Kawase T, et al. Gut microbiota of mice putatively modifies amino acid metabolism in the host brain. Br J Nutr. 2017;117(6):775-783.

93. Sasaki T, et al. Control of appetite and food preference by NMDA receptor and its co-agonist d-serine. Int J Mol Sci. 2016;17(7):1081.

94. Savignac HM, et al. Prebiotic feeding elevates central brain derived neurotrophic factor, $\mathrm{N}$-methyl-D-aspartate receptor subunits and D-serine. Neurochem Int. 2013;63(8):756-764.

95. Gronier B, et al. Increased cortical neuronal responses to NMDA and improved attentional set-shifting performance in rats following prebiotic (B-GOS $\left.{ }^{\circledast}\right)$ ingestion. Eur Neuropsychopharmacol. 2018;28(1):211-224.
96. Kelley AE. Ventral striatal control of appetitive motivation: role in ingestive behavior and reward-related learning. Neurosci Biobehav Rev. 2004;27(8):765-776.

97. Sharp WG, et al. Successful pharmacotherapy for the treatment of severe feeding aversion with mechanistic insights from cross-species neuronal remodeling. Transl Psychiatry. 2017;7(6):e1157.

98. Liu J-J, et al. High on food: the interaction between the neural circuits for feeding and for reward. Front Biol (Beijing). 2015;10(2):165-176.

99. Farooqi IS, et al. Leptin regulates striatal regions and human eating behavior. Science. 2007;317(5843):1355.

100.Contreras-Rodríguez O, et al. Ventral and dorsal striatum networks in obesity: link to food craving and weight gain. Biol Psychiatry. 2017;81(9):789-796

101. Meye FJ, Adan RAH. Feelings about food: the ventral tegmental area in food reward and emotional eating. Trends Pharmacol Sci. 2014;35(1):31-40.

102. Liu S, Borgland SL. Regulation of the mesolimbic dopamine circuit by feeding peptides. Neuroscience. 2015;289:19-42.

103. González-Arancibia C, et al. Do your gut microbes affect your brain dopamine? Psychopharmacology (Berl). 2019;236(5):1611-1622.

104. Strandwitz P. Neurotransmitter modulation by the gut microbiota. Brain Res. 2018;1693(1):128-133.

105. Crumeyrolle-Arias M, et al. Absence of the gut microbiota enhances anxiety-like behavior and neuroendocrine response to acute stress in rats. Psychoneuroendocrinology. 2014;42:207-217.

106. Aarts E, et al. Gut microbiome in ADHD and its relation to neural reward anticipation. PLoS One. 2017;12(9):e0183509.

107. Jadhav KS, et al. Gut microbiome correlates with altered striatal dopamine receptor expression in a model of compulsive alcohol seeking. Neuropharmacology. 2018;141:249-259.

108. Buffington SA, et al. Microbial reconstitution reverses maternal diet-induced social and synaptic deficits in offspring. Cell. 2016;165(7):1762-1775.

109. Sgritta M, et al. Mechanisms underlying microbial-mediated changes in social behavior in mouse models of autism spectrum disorder. Neuron. 2019;101(2):246-259.

110. Soto M, et al. Gut microbiota modulate neurobehavior through changes in brain insulin sensitivity and metabolism. Mol Psychiatry. 2018;23(12):2287-2301.

111. Delbès AS, et al. Prebiotics supplementation impact on the reinforcing and motivational aspect of feeding. Front Endocrinol (Lausanne). 2018;9:273.

112. Arentsen T, et al. The bacterial peptidoglycan-sensing molecule Pglyrp2 modulates brain development and behavior. Mol Psychiatry. 2017;22(2):257-266.

113. Osadchiy V, et al. Correlation of tryptophan metabolites with connectivity of extended central reward network in healthy subjects. PLoS One. 2018;13(8):1-16

114. Byrne CS, et al. Increased colonic propionate reduces anticipatory reward responses in the human striatum to high-energy foods. Am JClin 
Nutr. 2016;104(1):5-14.

115. Stevenson RJ, Francis HM. The hippocampus and the regulation of human food intake. Psychol Bull. 2017;143(10):1011-1032.

116. Azevedo EP, et al. A role of Drd2 hippocampal neurons in context-dependent food intake. Neuron. 2019;102(4):873-886.

117. Yang AK, et al. Hippocampal input to the nucleus accumbens shell enhances food palatability. Biol Psychiatry. 2020;87(7):597-608.

118. Parcet MA, et al. Individual differences in hippocampal volume as a function of BMI and reward sensitivity. Front Behav Neurosci. 2020;14:53.

119. Mestre ZL, et al. Hippocampal atrophy and altered brain responses to pleasant tastes among obese compared with healthy weight children. Int JObes (Lond). 2017;41(10):1496-1502.

120. Mestre Z, et al. Associations between body weight, hippocampal volume, and tissue signal intensity in 12- to 18-year-olds. Obesity (Silver Spring). 2020;28(7):1325-1331.

121. Luczynski P, et al. Adult microbiota-deficient mice have distinct dendritic morphological changes: differential effects in the amygdala and hippocampus. Eur J Neurosci. 2016;44(9):2654-2666.

122. Heberden C. Modulating adult neurogenesis through dietary interventions. Nutr Res Rev. 2016;29(2):163-171.

123. Möhle L, et al. Ly6Chi monocytes provide a link between antibiotic-induced changes in gut microbiota and adult hippocampal neurogenesis. Cell Rep. 2016;15(9):1945-1956.

124. Ogbonnaya ES, et al. Adult hippocampal neurogenesis is regulated by the microbiome. Biol Psychiatry. 2015;78(4):e7-e9.

125. Erny D, et al. Host microbiota constantly control maturation and function of microglia in the CNS. Nat Neurosci. 2015;18(7):965-977.

126. Luck B, et al. Bifidobacteria shape host neural circuits during postnatal development by promoting synapse formation and microglial function. $S c i$ Rep. 2020;10(1):1-18.

127. Fröhlich EE, et al. Cognitive impairment by antibiotic-induced gut dysbiosis: analysis of gut microbiota-brain communication. Brain Behav Immun. 2016;56:140-155.

128. Bistoletti M, et al. Antibiotic treatment-induced dysbiosis differently affects BDNF and TrkB expression in the brain and in the gut of juvenile mice. PLoS One. 2019;14(2):1-20.

129. Clarke G, et al. The microbiome-gut-brain axis during early life regulates the hippocampal serotonergic system in a sex-dependent manner. Mol Psychiatry. 2013;18(6):666-673.

130. Janik R, et al. Magnetic resonance spectroscopy reveals oral Lactobacillus promotion of increases in brain GABA, $\mathrm{N}$-acetyl aspartate and glutamate. Neuroimage. 2016;125:988-995.

131. Tang W, et al. Roles of gut microbiota in the regulation of hippocampal plasticity, inflammation, and hippocampus-dependent behaviors. Front Cell Infect Microbiol. 2021;10:611014.

132. Vorhees CV, Williams MT. Assessing spatial learning and memory in rodents. ILAR J. 2014;55(2):310-332.

133. Antunes M, Biala G. The novel object recognition memory: neurobiology, test procedure, and its modifications. Cogn Process. 2012;13(2):93-110.

134.Fellini L, Morellini F. Mice create whatwhere-when hippocampus-dependent memories of unique experiences. J Neurosci. 2013;33(3):1038-1043.

135. McLean FH, et al. Rapid and reversible impairment of episodic memory by a high-fat diet in mice. Sci Rep. 2018;8(1):1-9.

136.Arnoriaga-Rodríguez $\mathrm{M}$, et al. Obesity impairs short-term and working memory through gut microbial metabolism of aromatic amino acids. Cell Metab. 2020;32(4):548-560.

137. Yang Y, et al. Early-life high-fat diet-induced obesity programs hippocampal development and cognitive functions via regulation of gut commensal Akkermansia muciniphila. Neuropsychopharmacology. 2019;44(12):2054-2064.

138. Beilharz JE, et al. Cafeteria diet and probiotic therapy: cross talk among memory, neuroplasticity, serotonin receptors and gut microbiota in the rat. Mol Psychiatry. 2018;23(2):351-361.

139. Andermann ML, Lowell BB. Toward a wiring diagram understanding of appetite control. Neuron. 2017;95(4):757-778.

140.Williams EKK, et al. Sensory neurons that detect stretch and nutrients in the digestive system. Cell. 2016;166(1):209-221.

141. Kaelberer MM, et al. A gut-brain neural circuit for nutrient sensory transduction. Science. 2018;361(6408):eaat5236.

142. Bellono NW, et al. Enterochromaffin cells are gut chemosensors that couple to sensory neural pathways. Cell. 2017;170(1):185-198.

143. Bai L, et al. Genetic identification of vagal sensory neurons that control feeding. Cell. 2019;179(5):1129-1143.

144.Kim DY, et al. A neural circuit mechanism for mechanosensory feedback control of ingestion. Nature. 2020;580(7803):376-380.

145. Chen J, et al. A vagal-NTS neural pathway that stimulates feeding. Curr Biol. 2020;30(20):3986-3998.

146. Han W, et al. A neural circuit for gut-induced reward. Cell. 2018;175(3):665-678.

147. Tellez LA, et al. A gut lipid messenger links excess dietary fat to dopamine deficiency. Science. 2013;341(6147):800-802.

148.Suarez AN, et al. Gut vagal sensory signaling regulates hippocampus function through multiorder pathways. Nat Commun. 2018;9(1):2181.

149. O'Leary OF, et al. The vagus nerve modulate BDNF expression and neurogenesis in the hippocampus. Eur Neuropsychopharmacol. 2018;28(2):307-316

150. de Lartigue G, Diepenbroek C. Novel developments in vagal afferent nutrient sensing and its role in energy homeostasis. Curr Opin Pharmacol. 2016;31:38-43.

151. Tan HE, et al. The gut-brain axis mediates sugar preference. Nature. 2020;580(7804):511-516.

152. Fernandes $\mathrm{AB}$, et al. Postingestive modulation of food seeking depends on vagusmediated dopamine neuron activity. Neuron. 2020;106(5):778-788

153. Qu T, et al. On the roles of the duodenum and the vagus nerve in learned nutrient preferences. Appetite. 2019;139:145-151.

154. Kupari J, et al. An atlas of vagal sensory neurons and their molecular specialization. Cell Rep. 2019;27(8):2508-2523.

155. Egerod KL, et al. Profiling of G protein-coupled receptors in vagal afferents reveals novel gutto-brain sensing mechanisms. Mol Metab. 2018;12:62-75

156. Chen $\mathrm{H}$, et al. A forward chemical genetic screen reveals gut microbiota metabolites that modulate host physiology. Cell. 2019;177(5):1217-1231.

157. Colosimo DA, et al. Mapping interactions of microbial metabolites with human G-protein-coupled receptors. Cell Host Microbe. 2019;26(2):273-282.

158. Dehhaghi M, et al. Microorganisms, tryptophan metabolism, and kynurenine pathway: a complex interconnected loop influencing human health status. Int J Tryptophan Res. 2019;12:1178646919852996.

159. Johnson EL, et al. Sphingolipids produced by gut bacteria enter host metabolic pathways impacting ceramide levels. Nat Commun. 2020;11(1):2471.

160.Sugisawa E, et al. RNA sensing by gut Piezo1 is essential for systemic serotonin synthesis. Cell. 2020;182(3):609-624.

161. Koh A, Bäckhed F. From association to causality: the role of the gut microbiota and its functional products on host metabolism. Mol Cell. 2020;78(4):584-596.

162. Nøhr MK, et al. Expression of the short chain fatty acid receptor GPR41/FFAR3 in autonomic and somatic sensory ganglia. Neuroscience. 2015;290:126-137.

163. Goswami C, et al. Short-chain fatty acids suppress food intake by activating vagal afferent neurons. J Nutr Biochem. 2018;57:130-135.

164. Shackley M, et al. Short chain fatty acids enhance expression and activity of the umami taste receptor in enteroendocrine cells via a $\mathrm{G} \alpha_{\mathrm{i} / 0}$ pathway. Front Nutr. 2020;7:568991.

165. Tolhurst $\mathrm{G}$, et al. Short-chain fatty acids stimulate glucagon-like peptide-1 secretion via the G-protein-coupled receptor FFAR2. Diabetes. 2012;61(2):364-371.

166. Psichas A, et al. The short chain fatty acid propionate stimulates GLP-1 and PYY secretion via free fatty acid receptor $2 \mathrm{in}$ rodents. Int JObes (Lond) 2015;39(3):424-429.

167. Caengprasath $\mathrm{N}$, et al. Internalization-dependent free fatty acid receptor 2 signaling is essential for propionate-induced anorectic gut hormone release. iscience. 2020;23(9):101449.

168. Buckley MM, et al. Glucagon-like peptide-1 secreting L-cells coupled to sensory nerves translate microbial signals to the host rat nervous system. Front Cell Neurosci. 2020;14:95.

169. Wahlström A, et al. Intestinal crosstalk between bile acids and microbiota and its impact on host metabolism. Cell Metab. 2016;24(1):41-50.

170.Cawthon CR, de La Serre CB. Gut bacteria interaction with vagal afferents. Brain Res. 2018;1693(Pt B):134-139.

171. de La Serre CB, et al. Chronic exposure to low dose bacterial lipopolysaccharide inhibits leptin signaling in vagal afferent neurons. Physiol Behav. 2015;139:188-194.

172. de Lartigue $G$, et al. Leptin resistance in vagal afferent neurons inhibits cholecystokinin signal- 
ing and satiation in diet induced obese rats. PLOS One. 2012;7(3):e32967.

173. de Lartigue G, et al. Deletion of leptin signaling in vagal afferent neurons results in hyperphagia and obesity. Mol Metab. 2014;3(6):595-607.
174. Sen T, et al. Diet-driven microbiota dysbiosis is associated with vagal remodeling and obesity. Physiol Behav. 2017;173:305-317.

175. Pelot NA, Grill WM. Effects of vagal neuromodulation on feeding behavior. Brain Res. 2018;1693(pt B):180-187.

176. Timshel PN, et al. Genetic mapping of etiologic brain cell types for obesity. Elife. 2020;9:e55851.

177. Leng G, et al. The determinants of food choice. Proc Nutr Soc. 2017;76(3):316-327. 